Pacific Journal of Mathematics

PRIME RINGS WITH A ONE-SIDED IDEAL SATISFYING A 


\title{
PRIME RINGS WITH A ONE-SIDED IDEAL SATISFYING
}

\section{A POLYNOMIAL IDENTITY}

\author{
L. P. Belluce and S. K. Jain
}

It is known that the existence of a nonzero commutative one-sided ideal in a prime ring implies that the whole ring is commutative. Since rings satisfying a polynomial identity are natural generalizations of commutative rings the question arises as to what extent the above mentioned result can be extended to include these generalizations. That is, if $R$ is a prime ring and $I$ a nonzero one-sided ideal which satisfies a polynomial identity does $R$ satisfy a polynomial identity?

This paper initiates an investigation of this problem. A counter example, given later, will show that the answer to the above question may be negative, even when $R$ is a simple primitive ring with nonzero socle. The main theorem of this paper is Theorem 3 which states:

Let $R$ be a prime ring having a nonzero right ideal which satisfies a polynomial identity. Then, a necessary and sufficient condition that $R$ satisfy a polynomial identity is that $R$ have zero right singular ideal and $\hat{R}$, the right quotient ring of $R$, have at most finitely many orthogonal idempotents.

2. In the following given a ring $R, R^{\Delta}\left({ }^{4} R\right)$ denotes the right (left) singular ideal of $R$. Thus $R^{s}=\left\{x \mid x \in R, x^{r} \in L^{\Delta}(R)\right\}$ where $L^{\Delta}(R)$ denotes the set of right ideals of $R$ that meet, in a nonzero fashion, all right ideals of $R$. Similarly for ${ }^{4} R$ and ${ }^{\Delta} L(R)$.

If $Q$ is a ring such that $R$ is a subring of $Q$ and $q R \cap R \neq 0$ for each $q \in Q$ then $Q$ is called a right quotient ring for $R$. Moreover if $Q=\left\{a b^{-1} \mid a, b \in R, b\right.$ regular $\}$ then $Q$ is called a classical right quotient ring. Following [2] we say that a ring $R$ is right quotient simple if and only if it has a classical right quotient ring $Q$ with $Q \cong D_{n}, D_{n}$ a ring of $n \times n$ matrices over a division ring $D$.

From [4] we know that if $R$ is a prime ring with $R^{\Delta}=0$ then $R$ has a unique maximal right quotient ring $\hat{R}$ where $\hat{R}$ is a prime regular ring. Moreover, letting $L(R)$ denote the lattice of right ideals of $R$, there is a mapping $s: A \rightarrow A^{s}$ of $L(R)$ which is a closure operation satisfying $0^{s}=0,(A \cap B)^{s}=A^{s} \cap B^{s}$ and $\left(x^{-1} A\right)^{s}=x^{-1} A^{s}$. The set $L^{s}(R)$ of closed ideals of $R$ can be made into a lattice in a natural way and it is shown in [4] that $L^{s}(R) \cong L^{s}(\hat{R})$ under the mapping $A \rightarrow A \cap R, A \in L^{s}(\hat{R})$. We shall have occasion to use the following realization of $\hat{R}$. Let $E=\cup_{\Delta \in L^{\Delta_{(}(R)}} \operatorname{Hom}_{R}(A, R)$. On $E$ 
define the relation, $\alpha \equiv \beta$ if for some $A \in L^{4}(R), A \subseteq \operatorname{Dom} \alpha \cap \operatorname{Dom} \beta$ and $\alpha(x)=\beta(x)$ for each $x \in A$. It is shown in [5] that $\equiv$ is an equivalence relation and that $E / \equiv$ is a ring and in fact is $\hat{R}$.

The above remarks apply similarly to a prime ring $R$ for which ${ }^{\Delta} R=0$.

3. In this section occur the basic results of this paper. We will have occasion to use the result of Posner [8] stating that if $R$ is a prime ring with polynomial identity then $\hat{R}$ is a classical twosided quotient ring having the same multilinear identities as $R$. That part of Posners argument that shows if $R$ has a polynomal identity then so does $\hat{R}$ is a very complicated argument and we take this opportunity to present a simple alternative argument.

LEMMA 1. Let $R$ be a prime ring with polynomial identity. Then $\hat{R}$ has a polynomial identity.

Proof. From Posner [8] we know that $R$ has left and right quotient conditions and hence $R$ is right quotient simple, with $\hat{R} \cong D_{n}$. By a theorem of Faith and Utumi [2] $R$ contains an integral domain $K$ with right quotient ring $\hat{K} \cong \hat{D}$. Since $K$ satisfies a polynomial identity we have by Amitsur [1] that $\hat{K}$ also has a polynomial identity. Thus $D$, and hence $D_{n}$, is finite dimensional over its center; thus $D_{n}$, so $\hat{R}$, has a standard identity.

Lemma 2. Let $R$ be a prime ring with $R^{A}=0$, let $A \in L^{A}(R)$ and let $\alpha \in \operatorname{Hom}_{R}(R, R), R$ considered as a right $R$-module. If $\alpha(A)=0$ then $\alpha=0$.

Proof. Let $x \in R$; then we have that $x^{-1} A \in L^{\Delta}(R)$. If $r \in x^{-1} A$ then $x r \in A$ and thus $\alpha(x r)=0$. Since $\alpha$ is a right $R$-endomorphism, $\alpha(x r)=\alpha(x) \cdot r:$ It follows that $\alpha(x) \cdot x^{-1} A=0$, hence $x^{-1} A \leqq \alpha(x)^{r}$. Thus $\alpha(x)^{r} \in L^{\Delta}(R)$ and so $\alpha(x) \in R^{4}$. Hence $\alpha(x)=0$.

The following lemma is trivial in the case $R$ contains a central element. Without a central element the proof is more involved.

LEMMA 3. Let $R$ be a prime ring with a polynomial identity. Then $\operatorname{Hom}_{R}(R, R)$ has a polynomial identity, if $R^{4}=0$.

Proof. From Lemma 1 we know that $\hat{R}$ has a polynomial identity. Consider $\hat{R}$ realized as $\cup_{A \in L(R)} \operatorname{Hom}_{R}(A, R) / \equiv$. For $\alpha \in \operatorname{Hom}_{R}(R, R)$ let $\bar{\alpha}$ denote the equivalence class in $\hat{R}$ determined by $\alpha$. The mapping $\alpha \rightarrow \bar{\alpha}$ is a homomorphism of $\operatorname{Hom}_{R}(R, R)$ into $\hat{R}$. If $\bar{\alpha}=\bar{\beta}$ then for 
some $A \in L^{\Delta}(R) \alpha(x)=\beta(x), x \in A$. Thus $(\alpha-\beta)(A)=0$. By Lemma 2 we see that $\alpha=\beta$. Thus $\alpha \rightarrow \bar{\alpha}$ is an injection onto a subring of $\hat{R}$ and so $\operatorname{Hom}_{R}(R, R)$ has a polynomial identity.

The following theorem provides a sufficient condition on the right ideal $I$ having a polynomial identity to ensure the whole ring has a polynomial identity.

THEOREM 1. Let $R$ be a prime ring having a right ideal $I \neq 0$, $I$ satisfying a polynomial identity and $I_{l}=0$. Then $R$ satisfies $a$ polynomial identity.

Proof. By assumption $I_{l}$, the left annihilator of $I$, is 0 . Hence $I$ is a prime ring itself. Considering $I$ as a left $I$-module we have by the obvious dual of Lemma 3 that $\operatorname{Hom}_{I}(I, I)$, (the left $I$-endomorphisms), has a polynomial identity. For $x \in R$ the mapping $x \rightarrow r_{x}$, right multiplication by $x$, is an anti-isomorphism of $R$ into $\operatorname{Hom}_{I}(I, I)$. Thus $R$ itself satisfies a polynomial identity.

THEOREM 2. Let $R$ be a right quotient simple ring, $I \neq 0$ a right ideal of $R$ satisfying a polynomial identity. Then $R$ satisfies a polynomial identity.

Proof. From Goldie [3] we have that $I$ contains a uniform right ideal, thus we may assume $I$ is uniform. Since $R^{\Delta}=0$ it follows that $\left\{x \mid x \in I, x^{r} \in L^{\lrcorner}(R)\right\}=0$, hence from [6] we have that $K=\operatorname{Hom}_{R}(I, I)$ is an integral domain. Moreover it is known ([3]) that $\hat{K} \cong D, D$ a division ring, where $\hat{R} \cong D_{n}$. To complete the proof it suffices to show that $D$ has a polynomial identity; the latter will hold provided $K$ has a polynomial identity. To this end consider the homomorphism $a \rightarrow l_{a}$, left multiplication by $a$, of $I$ into $K$. Let $J$ denote the image of this map. $J=0$ implies $I^{2}=0$ which is impossible; hence $J$ is a nonzero subring of $K$ satisfying a polynomial identity. Let $\alpha \in K$ and let $l_{a} \in J$. Let $x \in I$. Then $\alpha l_{\alpha}(x)=\alpha(a x)=\alpha(a) \cdot x=l_{\alpha(a)}(x)$. Thus $\alpha l_{a}=l_{\alpha(a)} \in J$. Hence $J$ is a left ideal of $K$. Since $K$ is an integral domain we have by an obvious dual to Theorem 1 that $K$ has a polynomial identity.

We now obtain, easily, the following.

THEOREM 3. Let $R$ be a prime ring having a nonzero right ideal which satisfies a polynomial identity. Then, a necessary and sufficient condition that $\hat{R}$ satisfy a polynomial identity is that $R^{\Lambda}=0$ and $\hat{R}$ have at most a finite number of orthogonal idempotents. 
Proof. Necessity is clear. Conversely, then, since $\hat{R}$ is regular with at most finitely many orthogonal idempotents it follows from [7] that $\hat{R}$ has the descending chain condition (d.c.c.) on right ideals. $\hat{R}$ is prime, thus $\hat{R} \cong D_{n}$ for some division ring $D$. Since $L^{s}(R) \cong L^{s}(\hat{R})$ we see that $L^{s}(R)$ has d.c.c. Thus from [4] we see that $\hat{R}$ is a classical right quotient ring, hence Theorem 2 applies.

The following example (communicated orally to $\mathrm{S}$. K. Jain by A. S. Amitsur) shows that the extension of an identity from a right ideal to the entire ring is not always possible. Let $F$ be a field and let $F_{\infty}$ be the ring of all infinite matrices of finite rank. Let $a=\left(A_{i j}\right)$ be a matrix such that $a_{11} \neq 0$ and $a_{i j}=0$ for $i, j \neq 1$. Let $I=a F_{\infty}$. Then $I$ satisfies the identity $(x y-y x)^{2}=0$ but $F_{\infty}$ satisfies no identity at all.

4. REMARKS. In the case that $R$ is primitive with a right ideal $I \neq 0$ having a polynomial identity then it is sufficient to assume that $R$ has at most a finite number of orthogonal idempotents to ensure that $R$ also have a polynomial identity.

There are other conditions one may impose upon $R$ and $I$ besides those given here, e.g. if $R$ has at most finitely many orthogonal idempotents and $I$ is a maximal right ideal or if $R^{A}=0$ and $I \in L^{4}(R)$.

\section{REFERENCES}

1. S. A. Amitsur, On rings with identities, J. London Math. Soc. 30 (1955), 464-470.

2. C. Faith and Y. Utumi, On Noetherian prime rings, Trans, Amer. Math. Soc. 114 (1965), 53-60.

3. A. W. Goldie, The structure of prime rings under ascending chain conditions, Proc. London Math. Soc. 8 (1958), 589-608.

4. R. E. Johnson, Quotient rings of rings with zero singular ideal, Pacific J. Math. 11 (1961), 1385-1392.

5. - The extended centralizer of a ring over a module, Proc. Amer. Math. Soc. 2 (1951), 891-89.

6. R. E. Johnson and E. T. Wong, Quasi-injective modules and irreducible rings, J. London Math. Soc. 36 (1961), 260-268.

7. I. Kaplansky, Topological representations of algebras. II, Trans. Amer. Math. Soc. 68 (1950), 62-75.

8. E. Posner, Prime rings satisfying a polynomial identity, Proc. Amer. Math. Soc. 11 (1960), 180-183.

Received October 9, 1964, and in revised form July 1965.

The second author was partially supported by NSF-Grant No. GP-1447.

University of CaLiForNia Riverside 


\section{PACIFIC JOURNAL OF MATHEMATICS}

\section{EDITORS}

\author{
H. ROYDEN \\ Stanford University \\ Stanford, California
}

\author{
J. P. JANS \\ University of Washington \\ Seattle, Washington 98105
}

J. DugundJI

Department of Mathematics

Rice University

Houston, Texas 77001

RICHARD ARENS

University of California

Los Angeles, California 90024

\section{ASSOCIATE EDITORS}
E. F. BECKENBACH
B. H. NeumanN
F. WOLF
K. YOSIDA

\section{SUPPORTING INSTITUTIONS}

\author{
UNIVERSITY OF BRITISH COLUMBIA \\ CALIFORNIA INSTITUTE OF TECHNOLOGY \\ UNIVERSITY OF CALIFORNIA \\ MONTANA STATE UNIVERSITY \\ UNIVERSITY OF NEVADA \\ NEW MEXICO STATE UNIVERSITY \\ OREGON STATE UNIVERSITY \\ UNIVERSITY OF OREGON \\ OSAKA UNIVERSITY \\ UNIVERSITY OF SOUTHERN CALIFORNIA
}

\author{
STANFORD UNIVERSITY \\ UNIVERSITY OF TOKYO \\ UNIVERSITY OF UTAH \\ WASHINGTON STATE UNIVERSITY \\ UNIVERSITY OF WASHINGTON \\ AMERICAN MATHEMATICAL SOCIETY \\ CHEVRON RESEARCH CORPORATION \\ TRW SYSTEMS \\ NAVAL ORDNANCE TEST STATION
}

\footnotetext{
Mathematical papers intended for publication in the Pacific Journal of Mathematics should be in typed form or offset-reproduced, double spaced with large margins. Underline Greek letters in red, German in green, and script in blue. The first paragraph or two must be capable of being used separately as a synopsis of the entire paper. It should not contain references to the bibliography. Manuscripts may be sent to any one of the four editors. All other communications to the editors should be addressed to the managing editor, Richard Arens, University of California, Los Angeles, California 90024.

Each author of each article receives 50 reprints free of charge; additional copies may be obtained at cost in multiples of 50 .

The Pacific Journal of Mathematics is published monthly. Effective with Volume 16 the price per volume (3 numbers) is $\$ 8.00$; single issues, $\$ 3.00$. Special price for current issues to individual faculty members of supporting institutions and to individual members of the American Mathematical Society: $\$ 4.00$ per volume; single issues $\$ 1.50$. Back numbers are available.

Subscriptions, orders for back numbers, and changes of address should be sent to Pacific Journal of Mathematics, 103 Highland Boulevard, Berkeley 8, California.

Printed at Kokusai Bunken Insatsusha (International Academic Printing Co., Ltd.), 7-17, Fujimi 2-chome, Chiyoda-ku, Tokyo, Japan.

PUBLISHED BY PACIFIC JOURNAL OF MATHEMATICS, A NON-PROFIT CORPORATION

The Supporting Institutions listed above contribute to the cost of publication of this Journal, but they are not owners of publishers and have no responsibility for its content or policies.
} 


\section{Pacific Journal of Mathematics}

\section{Vol. 24, No. $3 \quad$ July, 1968}

Duane W. Bailey, On symmetry in certain group algebras ............ 413

Lawrence Peter Belluce and Surender Kumar Jain, Prime rings with a one-sided ideal satisfying a polynomial identity ................ 421

L. Carlitz, A note on certain biorthogonal polynomials ............. 425

Charles O. Christenson and Richard Paul Osborne, Pointlike subsets of a manifold ......................................... 431

Russell James Egbert, Products and quotients of probabilistic metric

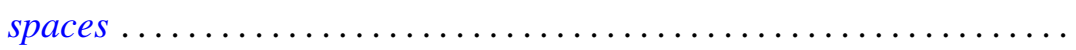

Moses Glasner, Richard Emanuel Katz and Mitsuru Nakai, Bisection into small annuli ..................................... 457

Karl Edwin Gustafson, A note on left multiplication of semigroup

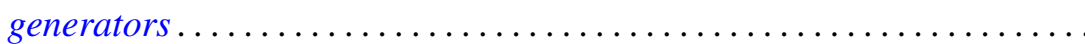

I. Martin (Irving) Isaacs and Donald Steven Passman, A characterization of groups in terms of the degrees of their characters. II ............. 467

Howard Wilson Lambert and Richard Benjamin Sher, Point-like

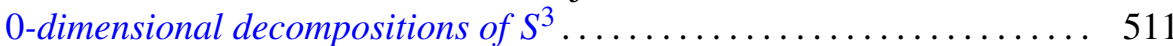

Oscar Tivis Nelson, Subdirect decompositions of lattices of width two ..... 519

Ralph Tyrrell Rockafellar, Integrals which are convex functionals . . . . . . . 525

James McLean Sloss, Reflection laws of systems of second order elliptic differential equations in two independent variables with constant coefficients ...

Bui An Ton, Nonlinear elliptic convolution equations of Wiener-Hopf type in a bounded region

Daniel Eliot Wulbert, Some complemented function spaces in $C(X)$

Zvi Ziegler, On the characterization of measures of the cone dual to a generalized convexity cone. 